\title{
Performance of europium aluminium doped polymer optical waveguide amplifier
}

\author{
Nur Najahatul Huda Saris ${ }^{1}$, Azura Hamzah ${ }^{2}$, Sumiaty Ambran $^{3}$, Osamu Mikami ${ }^{4}$, Takaki Ishigure ${ }^{5}$ \\ ${ }^{1,2,3,4}$ Department of Electronic Systems Engineering, Malaysia-Japan International Institute of Technology, Universiti \\ Teknologi Malaysia, 54100, Kuala Lumpur, Malaysia \\ ${ }^{5}$ Faculty of Science and Technology, Keio University, 3-14-1 Hiyoshi, Kohoku-ku, Yokohama, 223-8522, Japan
}

\begin{tabular}{l} 
Article Info \\
\hline Article history: \\
Received Mar 29, 2019 \\
Revised Jun 2, 2019 \\
Accepted Jun 28, 2019 \\
\hline
\end{tabular}

\section{Keywords:}

Europium aluminium

Forward pumping

Mosquito method

Optical amplifier

Polymer waveguide

\begin{abstract}
In this paper, the graded index (GI) multimode rare-earth metal (RE-M) doped polymer optical waveguide amplifier has been prepared and tested optically. A 10-cm Europium Aluminum Benzyl Methacrylate $\left(\mathrm{EuAl}_{3} \mathrm{O}_{3} / \mathrm{BzMA}\right)$ was fabricated via a unique technique known as the "Mosquito Method" which utilizes a micro-dispenser machine. Optical gain from 75 to $150 \mu \mathrm{m}$ circular core diameter waveguide of $13 \mathrm{wt} \%$ concentration has been demonstrated and measured under forward pumping condition. The cladding monomer deployed in this research is Acrylate resin XCL01, which is a modified photocurable acrylate material. Fundamentally, -30 decibel $(\mathrm{dBm})$ red light signal input and $23 \mathrm{dBm}$ pump power of $532 \mathrm{~nm}$ green laser wavelength is implemented within the range of 580 to $640 \mathrm{~nm}$ optical amplification wavelength. A maximum gain of $12.96 \mathrm{~dB}$ at $617 \mathrm{~nm}$ wavelength has been obtained for a $100 \mu \mathrm{m}$ core diameter of Eu-Al polymer optical waveguide. The effect of different coupler diameter for pumping and the comparison of insertion loss before and after amplification against the performance of the Eu-Al polymer waveguide amplifier are also studied. There exists an optimum core diameter of which the amplifier gain enhancement is at maximum value.
\end{abstract}

Copyright (C) 2019 Institute of Advanced Engineering and Science. All rights reserved.

\section{Corresponding Author:}

Nur Najahatul Huda Saris,

Department of Electronic Systems Engineering,

Universiti Teknologi Malaysia,

54100, Kuala Lumpur, Malaysia.

Email: nnhuda3@live.utm.my

\section{INTRODUCTION}

Over the past few years, utilization of lanthanide groups such as Terbium $\left(\mathrm{Tb}^{3+}\right)$, Erbium $\left(\mathrm{Er}^{3+}\right)$, Neodymium $\left(\mathrm{Nd}^{3+}\right)$ and Europium $\left(\mathrm{Eu}^{3+}\right)$ in lasers and amplifiers has grown substantially [1-3]. This is generally due to the effective pumping system of lanthanide ions' energy transfer process. Furthermore, lanthanide ions dissolve easily in organic hosts such as polymer by encapsulating it with organic ligand [4]. With that, higher doping concentration can be obtained. On top of that, research activities related to polymer optical fiber (POF) with low-loss visible wavelength window for short distance communications have increased recently $[5,6]$. This is due to its advantages of having low production costs and processing flexibility for polymer over inorganic materials such as glass and crystal [7,8]. Polymer generally exhibits low absorption losses in the visible wavelength region $[9,10]$.

Therefore, for the development of organic-dye-doped polymeric devices such as rare earth (RE) doped polymer amplifier as integrated optical waveguide devices, scaling down the size to be compact is necessary. However, this may result in interaction between ions due to high doping concentration in the active layer [11]. The transition lifetime at the metastable state will increase as well, owing to insufficient energy of the ligand surrounding the RE ion [12]. Hence, the Europium-Aluminium (Eu-Al) polymer optical 
waveguide amplifier, which is a product of rare earth metal (RE-M) doping composition, is introduced by KRI Inc.

In this research, the Europium Aluminium Benzyl Methacrylate $\left(\mathrm{EuAl}_{3} \mathrm{O}_{3} / \mathrm{BzMA}\right)$ is used as the core monomer. However, this core monomer is yet to be commercialized. The synthesis of $\mathrm{EuAl}_{3} \mathrm{O}_{3} / \mathrm{BzMA}$ is relatively similar to that of a previous research conducted by KRI Inc., concerning Europium Aluminium Methyl Methacrylate $\mathrm{EuAl}_{3} \mathrm{O}_{3}$ /MMA $[12,13]$. However, the core monomer must undergo some chemical modification by replacing the Methyl Methacrylate (MA) with Benzyl Methacrylate (BzMA) after the evaporation process of the Propylene Glycol $\alpha$-Monomethyl Ether (PGME). This modification is important in order to enhance the core properties with respect to this research [14].

In the next few years, more advanced technology will be developed correlating to the demand of higher data rate. Thus, it is imperative to implement the graded index (GI) multimode fiber since the fiber is a promising candidate for high bitrate transmissions in which it has the advantage of low propagation loss, low connection loss with GI multimode fiber (MMF) with wide misalignment tolerance, and low interchannel crosstalk even under a small pitch size [15]. Correspondingly, the "Mosquito Method" is adopted to create the GI circular core [16-19] of the Eu-Al polymer optical waveguide. The circular core is important as this research attempts to combine optical fiber characteristics to a planar waveguide. The optical amplification for GI multimode Eu-Al polymer optical waveguide amplifier has been reported to have satisfactory results as high as $7.1 \mathrm{~dB} / \mathrm{cm}$ for $10 \mathrm{wt} . \%$ concentration via variable stripe length (VSL) method [14] and $3.24 \mathrm{~dB} / \mathrm{cm}$ for $13 \mathrm{wt} . \%$ through forward pumping method [20]. Although higher optical amplification was obtained from the VSL method, the side surface excitation method seemed complicated to implement and was impractical for current application [20, 21]. Furthermore, both methods acquired core diameters of $100 \mu \mathrm{m}$ for 5.2 and $4.9 \mathrm{~cm}$. Thus, the study proceeds with $\mathrm{Eu}-\mathrm{Al}$ polymer optical waveguide amplifier at $13 \mathrm{wt} . \%$ concentration of the core monomer. Accordingly, the research would focus on the relationship of optical gain and core diameter. The core diameter of the waveguide is precisely controlled to $75,100,125$ and $150 \mu \mathrm{m}$ with a deviation of only a few micrometers and tested experimentally. The length of the waveguide is fixed by doubling the length of the waveguide in the previous research, i.e., approximately $10 \mathrm{~cm}$.

\section{FABRICATION METHOD}

The Eu-Al polymer optical waveguide amplifier was fabricated by using a unique method known as the Mosquito Method. It was performed by utilizing a micro-dispenser provided by Musashi Engineering Inc. Basically, a viscous liquid-state core monomer from a syringe connected to a dispenser was dispensed via a thin needle directly into a cladding monomer layer. The Acrylate resin, XCL01 and Europium Aluminum Benzyl Methacrylate $\left(\mathrm{EuAl}_{3} \mathrm{O}_{3} / \mathrm{BzMA}\right)$ were used as cladding and core, with refractive index of 1.501 and 1.51, respectively. The fabrication technique is illustrated in Figure 1.

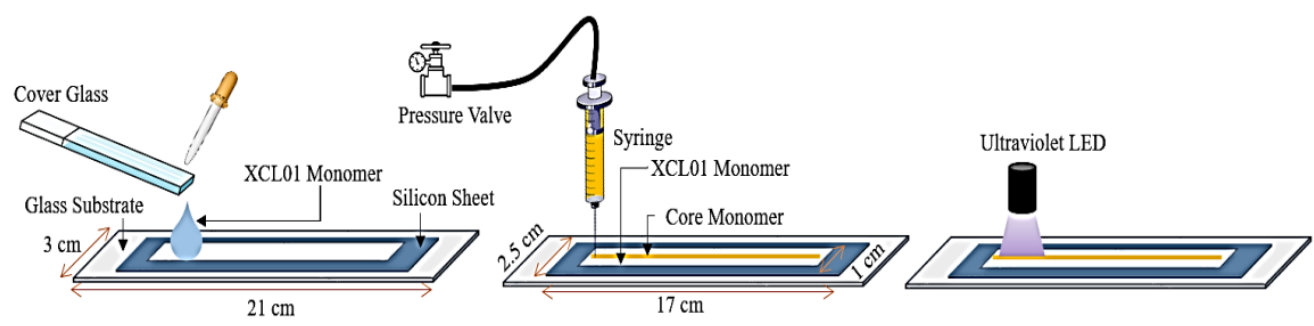

Figure 1. Fabrication steps of the mosquito method

Firstly, the XCL01 monomer was coated on the glass substrate. The waveguide material, which is the frame of cladding monomer was made from the silicone resins supplied by ADEKA Corp. to ensure a thick layer coating of cladding monomer. After that, $\mathrm{EuAl}_{3} \mathrm{O}_{3} / \mathrm{BzMA}$ was inserted into the syringe connected to the micro-dispenser. Then, $\mathrm{EuAl}_{3} \mathrm{O}_{3} / \mathrm{BzMA}$ was dispensed directly into the cladding layer through a thin needle tip attached to the syringe. Here, the dispensing scanning speed, pressure, and the needle inner diameter were scanned throughout the process. These parameters are the key factors that control the core diameter and inter-channel pitch [19, 22-25]. In the meantime, the $\mathrm{EuAl}_{3} \mathrm{O}_{3} / \mathrm{BzMA}$ was discharged directly onto a liquid state of XCL01. It is very important for the cladding in its liquid state to maintain the core's original cross-sectional shape immediately after being dispensed from the needle. Before exposure to ultra violet (UV) light, the core and cladding monomer should be diffused together since both monomers are miscible to form a concentration distribution. The copolymer was successfully formed after curing under UV 
light and postbaked such that the concentration distribution remained fixed. The waveguide was cut depending on the desired length and then it was ready for testing.

\section{RESULTS AND ANALYSIS}

In this section, the performance of rare earth metal (RE-M) using Europium Aluminum (Eu-Al) as gain medium is demonstrated with different core diameters. This section delineates the optimum parameters for dispensing conditions, experiment procedures and results, and data analyses. The performance of Eu-Al polymer optical waveguide is evaluated based on insertion loss and optical gain for a variation of core diameters with fixed length throughout the different coupler diameters used. The physical change on the surface of core waveguide are also discussed.

\subsection{Optimum dispensing conditions}

The dispensing scanning speed, inner needle diameter and dispensing pressure are key parameters in executing the Mosquito Method [16, 19, 22]. Hence, these three dispensing criteria are investigated to create the desired core diameter. In this research, the idea of the proposed optical waveguide amplifier is based on the combination of planar waveguide and graded index (GI) optical fiber core, upon realizing the superiority of the GI multimode fiber in high-speed transmissions.

For this purpose, three circular inner needle diameters (150, 170 and $190 \mu \mathrm{m})$ and dispensing gas pressure $(210$ and $420 \mathrm{kPa})$ were varied. The dependency of the core diameter on these three parameters are shown in Figure 2(a) and 2(b). The plots show the average of five core diameters created on the waveguide. From the results, the smaller cores were found to require faster dispensing scanning speed under smaller inner needle diameter of $150 \mu \mathrm{m}$ and low pressure of $210 \mathrm{kPa}$. In this paper, four waveguides with different core diameters of $75,100,125$, and $150 \mu \mathrm{m}$ were fabricated based on the appropriate dispensing conditions found for XCLO1 and $\mathrm{EuAl}_{3} \mathrm{O}_{3} / \mathrm{BzMA}$. Table 1 presents the fabrication results of desired core diameters according to manipulated and fixed parameters as set for each core diameter.

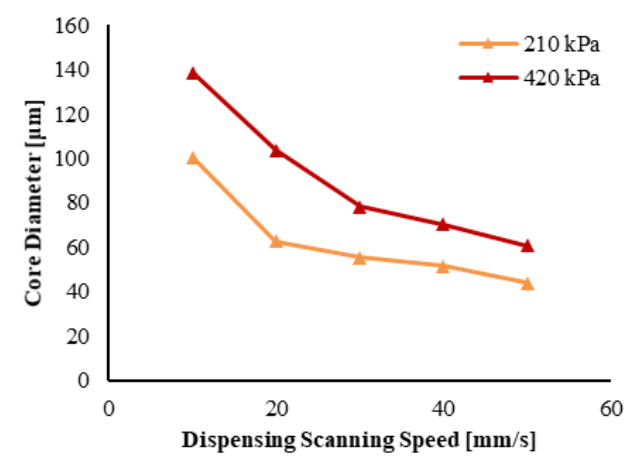

(a)

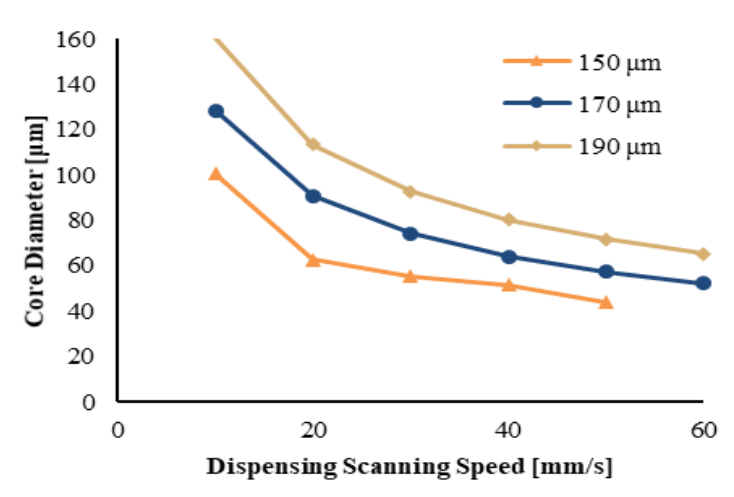

(b)

Figure 2. Core diameter versus dispensing scanning speed for variation of, (a) Dispensing pressure with $150 \mu \mathrm{m}$ inner needle diameter, and (b) Inner needle diameter with $210 \mathrm{kPa}$ dispensing pressure

Table 1. Parameters of micro dispenser machine

\begin{tabular}{cccc}
\hline Core diameter $[\mu \mathrm{m}]$ & The needle inner diameter $[\mu \mathrm{m}]$ & The dispenser gas pressure $[\mathrm{kPa}]$ & The needle scanning speed $[\mathrm{mm} / \mathrm{s}]$ \\
\hline 75 & 190 & 284 & 61.8 \\
100 & 190 & 505 & 61.8 \\
125 & 190 & 603 & 47.3 \\
150 & 190 & 505 & 27.5 \\
\hline
\end{tabular}

\subsection{Near field pattern and insertion loss}

Varying the waveguide core diameter is a very important aspect of this investigation in order to ascertain the optimum core diameter for best gain enhancement. Thus, each core from four waveguides was fabricated and tested multiple times at a wavelength of $617 \mathrm{~nm}$, which is one of the wavelengths under visible region. In this measurement, the near field pattern (NFP) is necessary for the optical beam pattern analysis. The NFP is used to analyze the light confinement from the transceiver side (Tx) to the Receiver side (Rx). In other words, by using the NFP, the position of the launching and receiving sides can be adjusted 
properly to prevent any loss during measurement. In this research, the input of the red-light signal was from a light emitting diode (LED) via single mode fiber (SMF) at the Tx side. Then, the light emitted through the waveguide was captured using a charge coupled device (CCD) camera at the Rx side as shown in Figure 3.

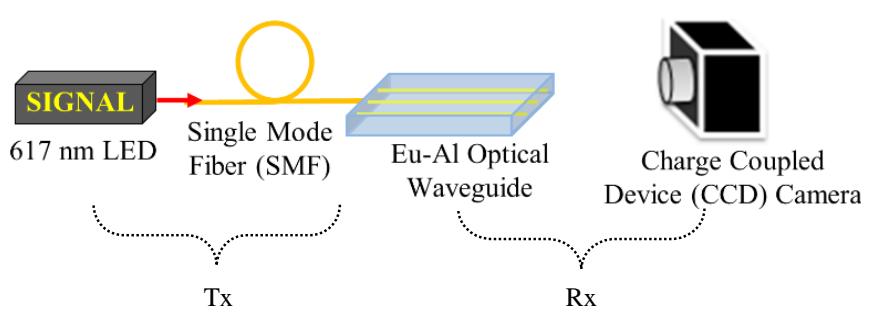

Figure 3. Near Field Pattern (NFP) measurement system

Next, two readings were taken into consideration for the insertion loss. The first reading was made from the configuration with the waveguide and measured as in Figure 4(a) and followed by the second reading of that the configuration without the waveguide, also known as "back to back" as in Figure 4(b). The output of the light was measured via a Power Meter along the fiber to determine the insertion loss. The core cross-section, NFP and insertion loss for the best core amplification are tabulated in Table 2.

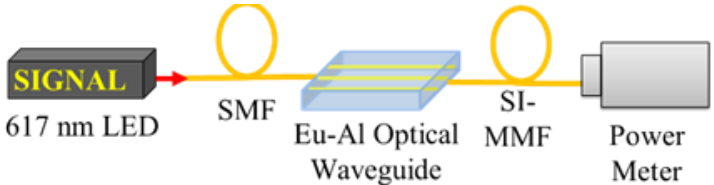

(a)

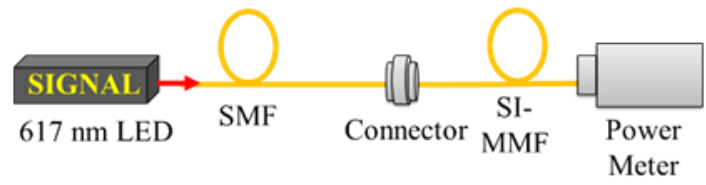

(b)

Figure 4. Insertion loss measurement, (a) Reading with waveguide, and (b) Back to back reading

Comparing the NFP measurement results in Table 2, a distinct colour distribution of the beam (light intensity distribution) is noticed. For example, the highest light distribution in $75 \mu \mathrm{m}$ core diameter waveguide is red, meanwhile, 100 and $150 \mu \mathrm{m}$ core diameter waveguides are blue. It is indicated that the red-light distribution in $75 \mu \mathrm{m}$ is more strongly confined to the core centre, whereas in other cores, it is considered that the lights spread out during propagation. This is believed to occur due to the difference of the refractive index distribution formed within the core. From these results, it is confirmed that light propagates through the core in any of the waveguides, although the manner in which the light is confined in the core centre is different between the waveguides.

Table 2. NFP and insertion loss results of different core diameters

\begin{tabular}{ccccc}
\hline Core Diameter $[\mu \mathrm{m}]$ & 75 & 100 & 125 & 150 \\
\hline & & & & \\
Core Cross-Section & & & & \\
& & & & \\
NFP & & & & \\
& & & & \\
& & & & \\
Back to Back $[\mathrm{dBm}]$ & -48.75 & -46.07 & -52.01 & -48.64 \\
Reading with & -40.57 & -40.81 & -40.75 & -40.98 \\
Waveguide $[\mathrm{dBm}]$ & 8.18 & 5.26 & 11.26 & 5.87 \\
Insertion Loss $[\mathrm{dBm}]$ & & & \\
\hline
\end{tabular}

The results for insertion loss measurements showed the highest insertion loss at $125 \mu \mathrm{m}$, which is $11.26 \mathrm{dBm}$. In contrast, the $75 \mu \mathrm{m}$ waveguide core diameter recorded the second lowest insertion loss, which 
is $8.18 \mathrm{dBm}$. Hence, it can be concluded that the reason for the instability of the insertion loss with respect to the waveguide core diameter is due to the polished state of the end face of the waveguide or measurement error.

\subsection{Gain enhancement for different coupler}

The experiment setup for optical amplification is shown in Figure 5. The green laser of $23 \mathrm{dBm}$ emitting at $532 \mathrm{~nm}$ wavelength was used as the pump source and $-30 \mathrm{~dB}$ red light signal emitting at $617 \mathrm{~nm}$ wavelength was used as the signal source. Both signal and light pump were combined using a $105 \mu \mathrm{m}$ core graded index-multimode fiber (GI-MMF) (50:50) coupler. The amplified output signal was collected by an optical spectrum analyzer (OSA) passing through two meters of $200 \mu \mathrm{m}$ core diameter of step index-multimode fiber (SI-MMF).

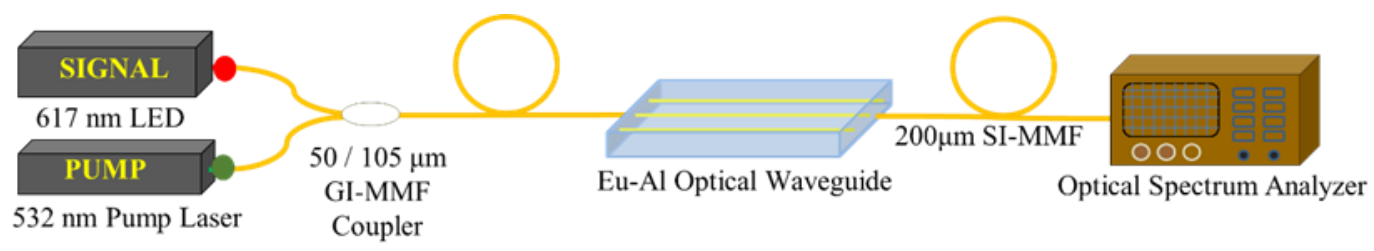

Figure 5. Optical gain amplification measurement setup

The repetitions of the gain measurement are necessary to investigate the repeatability and the resistance to high optical power excitation. The outputs from OSA were analyzed using Microsoft Excel. For this purpose, the measurement of the signal, pumping signal and the coupled signal of input and light pump were made separately. Consequently, the gain without any noise such as Amplified Spontaneous Emission could be obtained by using mathematical subtraction. Under this condition, the optical gain measurement was repeated by substituting the $105 \mu \mathrm{m}$ core with a $50 \mu \mathrm{m}$ core GI-MMF coupler.

Figure 6. shows the optical gain for different core diameters of the Eu-Al polymer optical waveguide amplifier using two different coupler diameters, which are 105 and $50 \mu \mathrm{m}$. From the graph, the optical gain is observed to have increased from 75 to $100 \mu \mathrm{m}$ waveguide core diameter for both couplers. For the $50 \mu \mathrm{m}$ coupler, the gain shows a slight decrease at 125 and $150 \mu \mathrm{m}$ waveguide core diameter, which are 11.93 and $8.93 \mathrm{~dB}$ respectively.

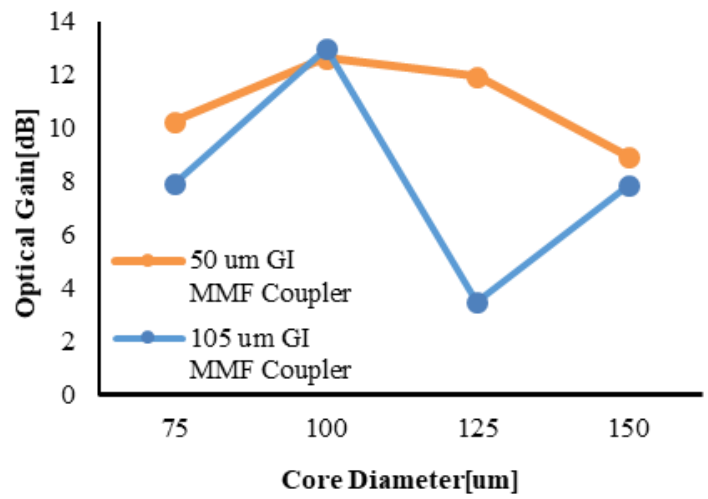

Figure 6. Comparison of Optical Gain for 105 and $50 \mu \mathrm{m}$ GI-MMF Coupler

However, the gain drastically decreases from 12.96 to $3.46 \mathrm{~dB}$ for the $105 \mu \mathrm{m}$ coupler at 100 to 125 $\mu \mathrm{m}$ waveguide core diameter. The decrease in gain value is believed to be due to measurement error. Surprisingly, the optical gain of $105 \mu \mathrm{m}$ coupler for $150 \mu \mathrm{m}$ waveguide core diameter increases sharply from $125 \mu \mathrm{m}$ which is $7.88 \mathrm{~dB}$. Overall, the trend of the optical amplification gain by using the $50 \mu \mathrm{m}$ coupler is higher compared to that of the $105 \mu \mathrm{m}$. This could be related to the higher energy density of the launching side provided by the $50 \mu \mathrm{m}$ coupler as compared to the $105 \mu \mathrm{m}$ coupler; i.e., $10.19 \times 10^{7}$ and $2.31 \times 10^{7} \mathrm{~W} / \mathrm{m}^{2}$ respectively, as proven from (1):

$$
\text { Energy Density }=\frac{\text { Power of Light }[\mathrm{W}]}{\text { Light Irradiation Area }\left[\mathrm{m}^{2}\right]}
$$

Performance of europium aluminium doped polymer optical waveguide... (Nur Najahatul Huda Saris) 
Although the gain attained by the $105 \mu \mathrm{m}$ coupler at $100 \mu \mathrm{m}$ is $12.96 \mathrm{~dB}$, which is higher compared to the $50 \mu \mathrm{m}$ coupler, the difference is not substantial, of which amounts to only $0.33 \mathrm{~dB}$ gap at the wavelength of $617 \mathrm{~nm}$.

\subsection{Comparison of insertion loss before and after amplification}

Table 3 compares the surface core cross-section before and after amplification. Clearly, the appearance from post-amplification generally shows a darker shade compared to pre-amplification. This could be attributed to higher heat emitted by the laser pump applied to the waveguide during amplification as supported by the measurement of insertion loss after the amplification in Figure 7.

As illustrated in the graph, the $125 \mu \mathrm{m}$ core recorded the highest increasing insertion loss after amplification, which is approximately $2.91 \mathrm{dBm}$ followed by the $100 \mu \mathrm{m}$ core of $1.73 \mathrm{dBm}$. Based on the graph, the insertion loss after the amplification increases for all core diameters except for the $75 \mu \mathrm{m}$ core diameter. The decrease in insertion loss value after the amplification is shown as $0.02 \mathrm{dBm}$. Even with decreasing values in insertion loss, the gap remains very close. As a result, it is thought that the value could be due to measurement error.

Table 3. Comparison of core cross section before and after amplification

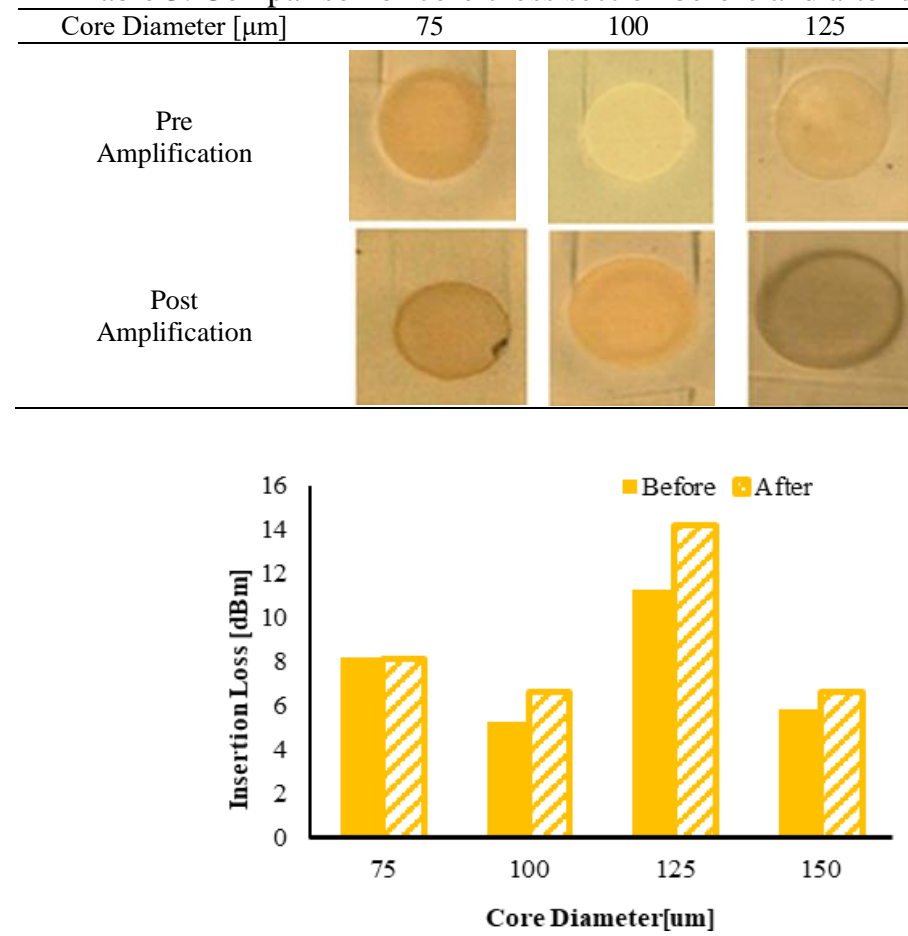

Figure 7. Insertion loss comparison before and after amplification

\section{CONCLUSION}

The Mosquito Method has been adopted in this study to prepare four different $10-\mathrm{cm}$ $\mathrm{EuAl}_{3} \mathrm{O}_{3}$ /BzMA polymer optical waveguides amplifiers with graded index multimode core diameters of 75, 100,125 , and $150 \mu \mathrm{m}$. It is confirmed that the fabrication of the desired core diameter using this method was dependent on the controllability of needle inner diameter, dispensing scanning speed and pressure. Optical amplification had been observed at $617 \mathrm{~nm}$ with a green laser at $532 \mathrm{~nm}$. Based on observation, the surface of the waveguide core turned darker-an effect from the heat emitted by the laser during amplification-which has caused the increase in insertion loss. Meanwhile, the highest gain recorded is 12.96 and $12.63 \mathrm{~dB}$ for $100 \mu \mathrm{m}$ waveguide core diameter using 105 and $50 \mu \mathrm{m}$ coupler respectively by forward pumping. The results show a potential for signal gain when incorporating $\mathrm{EuAl}_{3} \mathrm{O}_{3}$ /BzMA, a rare earth metal (RE-M) doped polymer, as an active optical device. Considerably, it is expected that this compact device could be integrated into many applications such as in-vehicle optical interconnect, medicine, and communication network in the near future. 


\section{ACKNOWLEDGEMENTS}

This research is a collaboration of Malaysia Japan International Institute of Technology (MJIIT) and Keio University and supported by Q.K130000.2543.17H26. We would like to show our gratitude to Keio University for their assistance in providing the facilities and instruments to conduct the research. We are also immensely grateful to Takaaki Ishigure Informatics and Optical Device System Laboratory members for their comments on earlier versions of the manuscript.

\section{REFERENCES}

[1] Slooff, L.H., et al., "Rare-earth doped polymers for planar optical amplifiers" Journal of Applied Physics, 2002. 91(7): p. 3955-3980.

[2] Zhou, B., et al., "Controlling upconversion nanocrystals for emerging applications", Nature Nanotechnology, 2015, 10(11): p. 924

[3] Johnson, N.J., et al., "Direct evidence for coupled surface and concentration quenching dynamics in lanthanide-doped nanocrystals", Journal of the American Chemical Society, 2017, 139(8): p. 3275-3282.

[4] Kuriki, K., Y. Koike, and Y. Okamoto, 'Plastic optical fiber lasers and amplifiers containing lanthanide complexes", Chemical Reviews, 2002. 102(6): p. 2347-2356.

[5] Tsang, K.C., C.-Y. Wong, and E.Y.B. Pun, "High-gain optical amplification in Eu 3+-doped polymer", Optics letters, 2010, 35(4): p. 520-522.

[6] Koike, Y. and K. Koike, "Progress in low-loss and high-bandwidth plastic optical fibers", Journal of Polymer Science Part B: Polymer Physics, 2011. 49(1): p. 2-17.

[7] Ribeiro, T., C. Baleizão, and J. Farinha, "Functional films from silica/polymer nanoparticles", Materials, 2014. 7(5): p. 3881-3900.

[8] Koike, Y. and A. Inoue, "High-speed graded-index plastic optical fibers and their simple interconnects for $4 \mathrm{~K} / 8 \mathrm{~K}$ video transmission", Journal of Lightwave Technology, 2016, 34(6): p. 1551-1555.

[9] Makino, K., et al., Low loss graded index polymer optical fiber with high stability under damp heat conditions. Optics express, 2012. 20(12): p. 12893-12898.

[10] Kaino, T., "Optical Absorption of Polymers", Encyclopedia of Polymeric Nanomaterials, S. Kobayashi and K. Müllen, Editors. 2014, Springer Berlin Heidelberg: Berlin, Heidelberg. p. 1-14.

[11] Koeppen, C., et al., Rare-earth organic complexes for amplification in polymer optical fibers and waveguides. JOSA B, 1997. 14(1): p. 155-162.

[12] Mataki, H., et al., "High-Gain Optical Amplification of Europium-Aluminum (Eu3+-Al)-Nanocluster-Doped Planar Polymer Waveguides", Japanese journal of applied physics, 2007, 46(1L): p. L83.

[13] H. Mataki and T. Fukui, "Organic/inorganic optical nanocomposite with highly-doped rare-earth nanoclusters: novel phosphors for white LEDs," 5th IEEE Conference on Nanotechnology, 2005., Nagoya, Japan, 2005, pp. $772-775$ vol. 2.

[14] Mitani, M., et al., "Polymer optical waveguide composed of europium-aluminum-acrylate composite core for compact optical amplifier and laser", Integrated Optics: Devices, Materials, and Technologies XIX, 2015, International Society for Optics and Photonics.

[15] Rasel, O.F., A. Yamauchi, and T. Ishigure, "Low-loss 3-dimensional shuffling graded-index polymer optical waveguides for optical printed circuit boards", IEICE Transactions on Electronics, 2018. 101(7): p. 509-517.

[16] Ishigure, T., "Graded-index core polymer optical waveguide for high-bandwidth-density optical printed circuit boards: fabrication and characterization", Optical Interconnects XIV, 2014, International Society for Optics and Photonics.

[17] S. Morikawa and T. Ishigure, "Fabrication of GI-core polymer optical waveguide using a dispenser and its application to optical printed circuit boards," 2010 23rd Annual Meeting of the IEEE Photonics Society, Denver, CO, 2010, pp. 108-109.

[18] Kinoshita, R., et al., "Polymer optical waveguides with GI and W-shaped cores for high-bandwidth-density on-board interconnects", Journal of Lightwave Technology, 2013, 31(24): p. 4004-4015.

[19] Kinoshita, R., D. Suganuma, and T. Ishigure, "Accurate interchannel pitch control in graded-index circular-core polymer parallel optical waveguide using the Mosquito method", Optics Express, 2014, 22(7): p. 8426-8437.

[20] Y. Yoshida, T. Fukui and T. Ishigure, "Polymer waveguide incorporated with europium-aluminum polymer composite for compact and high-gain optical amplification devices," 2017 Conference on Lasers and Electro-Optics Pacific Rim (CLEO-PR), Singapore, 2017, pp. 1-4.

[21] Y. Yoshida, T. Fukui and T. Ishigure, "Polymer waveguide incorporated with europium-aluminum polymer composite for compact and efficient amplification devices," 2017 IEEE CPMT Symposium Japan (ICSJ), Kyoto, 2017, pp. 181-184.

[22] Soma, K. and T. Ishigure, Fabrication of a graded-index circular-core polymer parallel optical waveguide using a microdispenser for a high-density optical printed circuit board. IEEE Journal of Selected Topics in Quantum Electronics, 2013, 19(2): p. 3600310-3600310.

[23] Takahashi, A. and T. Ishigure, "Bend insensitive graded index multimode polymer optical waveguides fabricated using the Mosquito method", Smart Photonic and Optoelectronic Integrated Circuits XVII. 2015, International Society for Optics and Photonics. 
[24] A. Yamauchi, Y. Morimoto, T. Enomoto, K. Suzuki, H. Masuda and T. Ishigure, "Graded-Index Multimode Polymer Optical Waveguide Enabling Low Loss and High Density 3D On-Board Integration," 2016 IEEE 66th Electronic Components and Technology Conference (ECTC), Las Vegas, NV, 2016, pp. 490-496.

[25] K. Soma and T. Ishigure, "Fabrication of polymer parallel optical waveguide with GI-circular-core using dispenser," 2012 4th Electronic System-Integration Technology Conference, Amsterdam, Netherlands, 2012, pp. 1-6.

\section{BIOGRAPHIES OF AUTHORS}
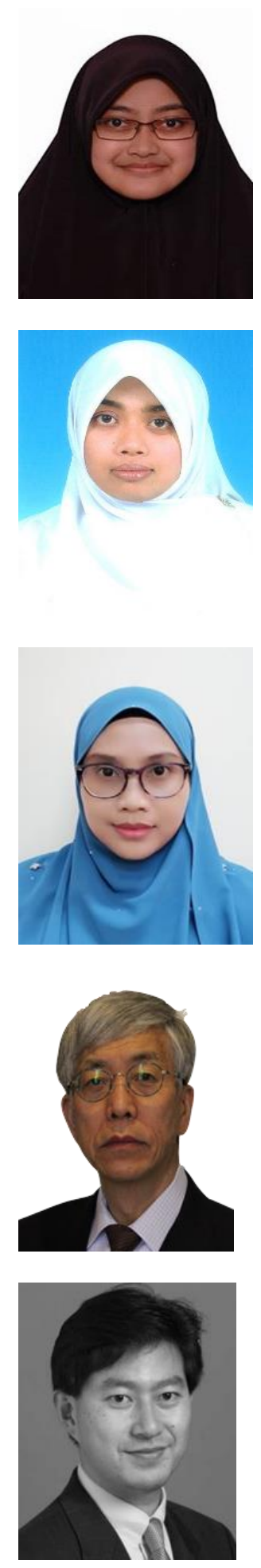

Takaaki Ishigure (M’00) was born in Gifu, Japan, on July 30, 1968. He received the B.S. degree in applied chemistry and the M.S. and Ph.D. degrees in material science from Keio University, Yokohama, Japan, in 1991, 1993, and 1996, respectively. He is currently an Assistant Professor with Keio University. He has been, concurrently, a Group Leader of the Japan Science and Technology (JST) Agency Exploratory Research for Advanced Technology (ERATO)-Solution-Oriented Research for Science and Technology (SORST) project. In 2005, he stayed at the Department of Electrical Engineering, Columbia University, New York, NY, as a Visiting Research Scientist. His current research interests are in the preparation of high-bandwidth graded-index plastic optical fibers (GI POF) and their system design. 